\title{
EMPLEO DE TÉCNICAS DE TELEDETECCIÓN DE MUY ALTA RESOLUCIÓN ESPACIAL PARA EL ANÁLISIS DEL COMPORTAMIENTO DE LAS VARIABLES FENOLÓGICAS EN CULTIVOS (PRUNUS PÉRSICA) CON RIEGO DEFICITARIO CONTROLADO
}

\author{
P. Pérez-Cutillas ${ }^{1,2}$ y J.J. Alarcón Cabañero ${ }^{2}$ \\ ${ }^{1}$ Universidad de Murcia \\ ${ }^{2}$ Centro de Edafología y Biología Aplicada del Segura
}

\section{RESUMEN}

El trabajo se realizó en una parcela de melocotoneros donde se aplicaron tratamientos de riego diferentes, lo que generó distinto grado de déficit hídrico en los árboles estudiados. Esta variabilidad en la situación hídrica de los árboles fue medida en superficie por medio del potencial hídrico de tallo a mediodía ( $\Psi$ s), y desde el aire por medio de la captación de una imagen obtenida con una cámara multiesprectal que permitieron estimar los valores del espectro infrarrojo cercano (NIR) y del índice de vegetación de diferencia normalizada (NDVI) con una resolución espacial de $25 \mathrm{~cm}$. Los índices obtenidos fueron sensibles al grado de comportamiento de las variables fenológicas de los cultivos tomadas en campo, que presentaron significativos valores de correlación con los valores obtenidos mediante técnicas de teledetección.

Palabras clave: agricultura de precisión, teledetección, sequía, relaciones hídricas, cultivos frutales.

\section{USING REMOTE SENSING TECHNIQUES OF VERY HIGH SPATIAL RESOLUTION ON PHENOLOGICAL BEHAVIOUR OVER CROPS IN REGULATED DEFICIT IRRIGATION}

\section{ABSTRACT}

Research focused on a peach orchard where different irrigation treatments were applied, inducing different degree of water stress in the trees. This water status variability was measured

Fecha de recepción: 28 de Marzo de 2014. Fecha de aceptación: 25 de Noviembre de 2014.

1 Departamento de Geografía. Universidad de Murcia pedrope@um.es

2 Centro de Edafología y Biología Aplicada del Segura. Consejo Superior de Investigaciones Científicas (CEBAS-CSIC).jalarcon@cebas.csic.es 
at field level by the use of stem water potential at midday ( $\left.\Psi_{\mathrm{s}}\right)$, and from remote sensing by the estimation of two parameters related to the analysis of high-resolution near-infrared images (NIR and NDVI) with $25 \mathrm{~cm}$ of spatial resolution. Indexes obtained were sensitive to the level of phenological behaviour of variables taken in field crops, which showed significant correlation values with the values obtained using remote sensing techniques.

Key words: precision farming, remote sensing, drought, water relations, fruit crops.

\section{INTRODUCCIÓN}

La agricultura en la Cuenca del Segura se encuentra entre las más rentables de España. En la Región de Murcia, que representa la mayoría de los cultivos de regadío de esta cuenca, aporta el $75 \%$ de la producción final. Pero la escasez de agua en esta zona provoca periódicamente elevadas pérdidas que afectan de manera notable a la economía (ARCAS Y HERNÁNDEZ, 2010). Los sistemas de explotación agrícolas tradicionalmente se ha visto influenciados por los distintos elementos climáticos, siendo la sequía uno de los que más afecta de forma perniciosa a la producción y la calidad de los productos agroalimentarios (KOGAN, 1997), especialmente en el sureste peninsular, que se caracteriza por la existencia de un clima semiárido y una escasa disponibilidad hídrica (GINESTAR Y CASTEL, 1996; GARRIDO et al., 2006; BLANCO-GUTIÉRREZ et al., 2013).

La exposición de las plantas a un ambiente con restricciones hídricas durante varios estadios de su desarrollo hace activar múltiples cambios fisiológicos y bioquímicos en ellas (ARAUS, 2004). En ambientes semiáridos, donde las altas temperaturas se unen a la escasez de las precipitaciones, que además no están distribuidas de manera homogénea a lo largo del año, hacen que cultivos los comerciales no se puedan concebir sin el apoyo del riego artificial. A pesar de ello, atendiendo a estas condiciones climáticas, estos cultivos pueden verse con frecuencia sometidos a condiciones de déficit hídrico (LOUSSERT, 1992; AGUSTÍ, 2003). Desde el punto de vista fisiológico, el estrés hídrico en las plantas se manifiesta de varias maneras. Se inhibe el crecimiento vegetativo, observándose una disminución en el crecimiento de los brotes y en el desarrollo foliar y la disminución del perímetro del tronco (ORTUNO et al., 2004). En las hojas se produce un cierre de estomas, la curvatura de los bordes de la hoja hacia el haz y la defoliación parcial. Se reduce el potencial osmótico y la conductividad en los estomas pero sin producirse un ajuste osmótico sino un ajuste elástico en el que se incrementa la elasticidad de los tejidos ante la falta de agua (SAVE et al., 1995; RUIZ-SANCHEZ et al., 1997). Y si se alarga ostensiblemente estas condiciones, puede llevar a daños importantes y marchitamiento de hojas. También se ha comprobado que el estrés hídrico reduce la producción, observándose sus efectos más significativos durante los estados fenológicos de floración y cuajado y desarrollo del fruto (GINESTAR Y CASTEL, 1996).

La evolución climática a nivel global apunta a una agricultura de regadío que se llevará a cabo cada vez más en condiciones de escasez de agua, y la gestión del riego debe pasar por mejorar la producción por unidad de superficie para maximizar la producción por unidad de agua consumida (FERERES y SORIANO, 2007). Por ello se hace necesaria 
una mejor gestión en los sistemas de riego, mediante técnicas como el Riego Deficitario Controlado (RDC) (CHALMERS, 1989), basado en la idea de reducir los aportes hídricos en los periodos fenológicos en los que un déficit hídrico controlado no afecta sensiblemente a la producción y calidad de la cosecha y, por otra parte, de cubrir la demanda total de la planta durante el ciclo del cultivo. Estas técnicas constituyen la base para la elaboración de estrategias de riego que permiten ahorrar importantes cantidades de agua con el menor impacto posible sobre la producción y en el medio ambiente (ALARCÓN et al., 2006).

El potencial que ofrecen las imágenes obtenidas por teledetección proporciona a la agricultura de precisión información distribuida espacial y temporalmente con el fin de supervisar procesos agrícolas, y poder con ello evaluar con mayor precisión la densidad óptima de cultivo, estimar con mejores resultados los niveles de fertilización y predecir con mayor exactitud los volúmenes de producción (VANDEN HEUVEL, 1996). Estas técnicas de monitorización de la vegetación se basan en la medición de los valores de reflectividad de los cultivos estableciendo una relación directa entre las medidas efectuadas por los sensores aerotransportados y los parámetros biofísicos que caracterizan a las distintas cubiertas vegetales (GILABERT et al, 1997). Los índices de vegetación se presentan como parámetros calculados a partir de estos valores de reflectividad en zonas espectrales del rojo e infrarrojo cercano (580-680 y 760-900 nm respectivamente), permitiendo la extracción de información relacionada con la vegetación y minimizando la influencia de perturbaciones debidas a elementos como el suelo o las condiciones atmosféricas, obteniéndose con ellos el noventa porciento de la información relativa a la vegetación (BANNARI et al, 1995). En trabajos de más detalle (SUÁREZ et al., 2010; STAGAKIS et al. 2012), se han obtenido interesantes relaciones entre el estrés hídrico en cultivos usando medidas in situ con datos derivados de imágenes captadas por sensores remotos, siendo determinantes el aporte de información hiperespectral (DZIKITI et al, 2010; KIM et al, 2011) y/o térmica (BERNI et al., 2009) en estos análisis.

Atendiendo a estos criterios, el objetivo del trabajo fue evaluar los efectos que el riego deficitario controlado tiene sobre el comportamiento fenológico de árboles frutales, y la evolución de algunos parámetros obtenidos con sistemas de detección terrestre utilizando imágenes de muy alta resolución, con información espectral en longitudes de onda del visible e infrarrojo cercano. Esto nos puede facilitar la búsqueda de correlaciones entre los valores medidos en campo, con los obtenidos mediante teledetección a modo de poder profundizar en el conocimiento de las relaciones existentes entre ambas fuentes de información, ayudando con ello, a la mejora en los procesos de detección de los efectos del estrés hídrico en amplias zonas de cultivos agrícolas.

\section{2. ÁREA DE ESTUDIO}

La parcela se encuentra situada en Fuente Librilla, Mula (Murcia), localizada entre las coordenadas UTM: X 637885 Y $_{1} 4198320$ - X 638220 Y $_{2} 4198125$ de la zona 30N, referidas al sistema de referencia ETRS89 (Figura 1). Los valores medios climáticos registrados en esta área presentan una precipitación media anual de $350 \mathrm{~mm}$, con una estimación de evapotranspiración potencial de $900 \mathrm{~mm}$, por lo que se calcula un déficit hídrico medio anual de unos $550 \mathrm{~mm}$ (AEMET, 2010). 
FIGURA 1

Localización parcela experimental.

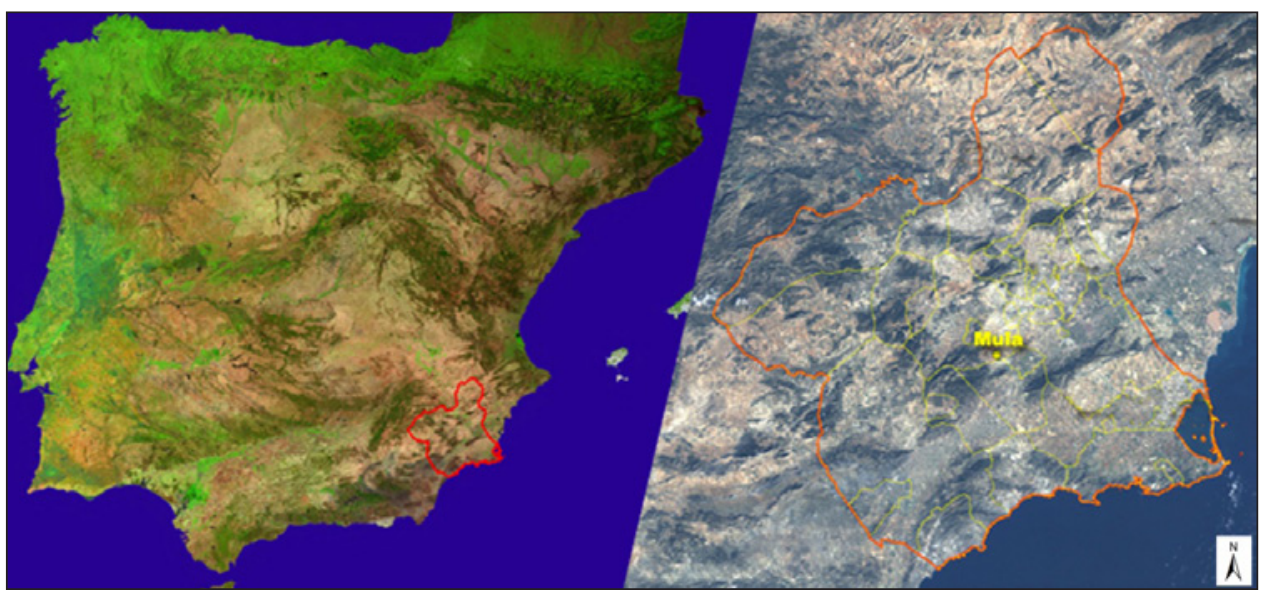

La altitud media del lugar donde está ubicada la parcela es de 374 metros, y una inclinación de aproximadamente 1 grado en la pendiente media. Los suelos se caracterizan por tener un régimen de humedad arídico y horizonte A ócrico muy débil. La unidad taxonómica a la que pertenece estos suelos, siguiendo el sistema FAO-UNESCO (1974), es de calcisoles háplicos, donde es muy frecuente la existencia de un horizonte petrocálcico (LUCDEME, 1986).

El ensayo fue llevado a cabo en una finca comercial sobre melocotoneros adultos (Prunus persica L. cv. 'Catherine') injertados sobre patrón GF677, con un marco de plantación 6 x $4 \mathrm{~m}$. Se aplicó riego por goteo, utilizándose un único lateral de riego por fila de árboles, con cinco goteros por planta, que arrojaban un caudal de $41 \mathrm{~h}-1$.

\section{MATERIAL Y MÉTODOS}

La parcela experimental se dividió equitativamente en tres tratamientos de riego: un tratamiento de riego control (C), que se regó satisfaciendo los requerimientos hídricos del cultivo (100\% ETc), y dos tratamientos de riego deficitario controlado (RDC) que recibieron respectivamente el $60 \%(\mathrm{RDCa})$ y $40 \%(\mathrm{RDCb})$ de agua que el tratamiento control. El período de RDC se extendió durante 35 días. Para la realización del ensayo se controlaron específicamente 21 árboles aleatoriamente distribuidos entre los diferentes tratamientos de riego (Figura 2).

Para las medidas de la situación hídrica a nivel de parcela, se midió el potencial hídrico de tallo a mediodía ( $\Psi_{\mathrm{S}}$ ) en hojas adultas, sanas y próximas al tronco envueltas en papel de aluminio y bolsa de plástico al menos dos horas antes de las medidas (3 hojas por árbol y tratamiento), para ello se empleó la cámara de presión (Soil Moisture Equip. Corp., modelo 3000 Santa Barbara CA, USA), según las indicaciones de HSIAO (1990).

Para la obtención de las imágenes del área de estudio se utilizó la cámara multiespectral ADS40 transportada en un avión tipo Partenavia P68C, obteniéndose una resolución 
FIGURA 2

Distribución de tratamientos y valores NDVI árboles controlados en la parcela experimental. Diseño experimental y vista aérea.

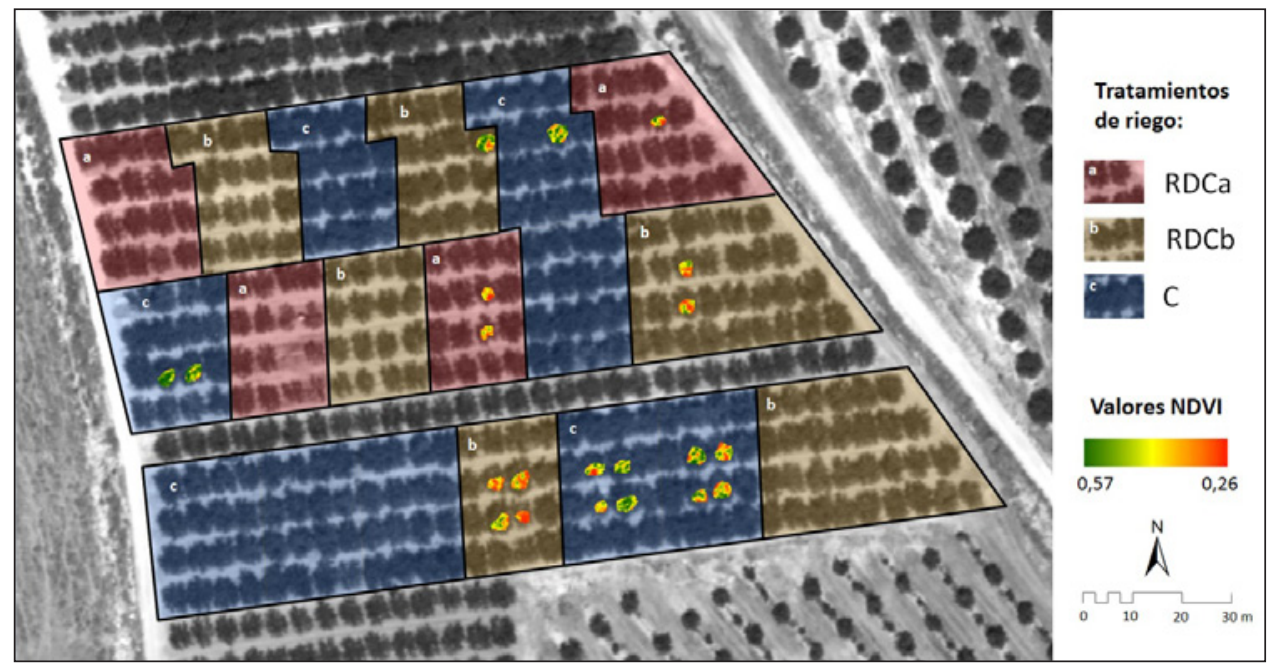

espacial para la imagen de $25 \mathrm{~cm}$ por píxel, una resolución radiométrica de 16 bits y una resolución espectral comprendida entre los 420 a los 900 nanómetros. Este vuelo se realizó el 01/09/2009, coincidiendo con la toma de datos en campo.

Es fundamental señalar la importancia de la calidad en la resolución radiométrica de la imagen en trabajos de este tipo, ya que un alto rango radiométrico de la imagen mejora la sensibilidad para expresar los datos recogidos por el sensor aumentando el número de Niveles Digitales (ND) utilizados ( 8 bits $=256$; 11 bits $=2048 ; 16$ bits $=65536$ valores) $(\mathrm{CHU}$ VIECO, 2002). De esta manera se mejoran los mecanismos de discriminación de detalle y permiten distinguir procesos que con menor nivel radiométrico serían imposibles observar.

Mediante un SIG se obtuvieron los datos pertenecientes al espectro visible e infrarrojo cercano (NIR). Para ello, se localizaron en la imagen todos los árboles seleccionados aleatoriamente con la ayuda de las coordenadas tomadas en campo por un GPS submétrico (Trimble GeoXT). Seguidamente se digitalizó el perímetro de todos los individuos para generar unas «mascaras» de corte, pero previamente se realizó una eliminación de los pixeles exteriores con el fin de minimizar el efecto borde y dejar fuera de los análisis los posibles «ruidos» que pudieran producir la superficie del suelo (LYCHAK y JAREMY. 2000), es decir, valores o información que no se correspondan estrictamente con lo que realmente buscábamos en los árboles en estudio.

Con estos elementos «mascara» mejor definidos, se inició el proceso de extracción de la información captada en las longitudes de onda del visible e infrarrojo cercano del espectro electromagnético, con las que se posibilita el cálculo de Índices de Vegetación como el NDVI (Índice de Vegetación de Diferencia Normalizada), índice de sobra conocido y acreditado por numerosos trabajos (CRIPPEN, 1990; CHUVIECO et al., 2001; 
MARTÍNEZ-BELTRÁN et al., 2009) donde se calcula el cociente de las diferencias entre el NIR, como reflectancia en el infrarrojo cercano y R, que es la reflectancia del rojo en la porción visible del espectro electromagnético. La particularidad de este índice es la alta correlación con la biomasa verde y la productividad primaria (ROUSE et al., 1974; GOWARD y HUEMMRICH, 1992).

Los datos analizados tanto en los algoritmos realizados para la obtención de los Índices de Vegetación utilizados en este estudio, así como los procesos estadísticos realizados, han sido elaborados a partir de los ND de las imágenes originales captadas por el sensor. La alta resolución espacial de las imágenes obtenidas ha permitido obtener una media de 86 pixeles por árbol, lo cual permite una amplia caracterización de la respuesta espectral para cada uno de los individuos.

\section{RESULTADOS Y DISCUSIÓN}

Los resultados obtenidos se han realizado mediante una serie de análisis estadísticos utilizando programas informáticos específicos: SPSS y lenguaje R.

Como ya se ha descrito en el apartado de material y métodos, el rango espectral de las imágenes utilizadas no permite obtener información del espectro térmico, ni el detalle de los sensores hiperespectrales. Esto nos determina que el estudio se enfoque hacia un análisis indirecto de los efectos del estrés hídrico a partir del comportamiento del estado fenológico de los frutales y su relación con los datos obtenidos en campo, respecto a los diferentes tratamientos de RDC.

En el experimento, el RDC afectó al potencial hídrico de tallo, de modo que los árboles sometidos a tratamiento Control siempre mostraron valores más elevados de potencial hídrico que aquellos sometidos a déficit hídrico. En la tabla de descriptivos de las muestras (Tabla 1) se puede observar como existe una tendencia directa entre los valores del potencial hídrico y la disponibilidad de agua con respecto al tratamiento Control ( $\mathrm{C}=-8,4 \mathrm{MPa})$, al Riego Controlado Deficitario bajo (RDCb= -17,2 $\mathrm{MPa}$ ) y al Riego Controlado Deficitario alto $(\mathrm{RDCa}=-19,8 \mathrm{MPa}$ ), tendencia que siguen las variables espectrales entre el tratamiento Control y los RDC, pero que no queda suficientemente clara entre las diferencias de RDCb y RDCa. Esto se puede deber a que la reducción del aporte hídrico en estos dos tratamientos deficitarios no son lo suficientemente dispares para poder ser captados por el sensor, lo que nos indica la existencia de ciertos umbrales por los cuales nos será imposible discriminar estas diferencias. Debido a estas limitaciones se plantea el posterior análisis estadístico con solo dos tratamientos, uno Control y otro RDC.

Debido a que los datos procedentes de los valores espectrales de las imágenes no siguen una distribución normal, para poder ser comparados con los distintos aportes de riego se ha realizado el test U de Mann-Whitney. Con esta prueba se han contrastado diferencias entre los dos tipos de aportes hídricos (control y deficitarios) que resultaron significativas para las variables potencial hídrico en tallo $\left(\Psi_{\mathrm{S}}\right.$ : $\left.\mathrm{U}=0.00 \mathrm{p}<0.05\right)$, para los valores del infrarrojo cercano (NIR: $\mathrm{U}=12.00, \mathrm{p}=0.002$ ), y para el índice de diferencia normalizada (NDVI: $\mathrm{U}=13.00, \mathrm{p}=0.002$ ). De este análisis han resultado significativas las diferencias para todas las variables consideradas ( $\left.\Psi_{\mathrm{S}}, \mathrm{NIR}, \mathrm{NDVI}\right)$ como muestra la tabla 2. 
TABLA 1

Valores descriptivos de las variables Potencial Hídrico $\left(\Psi_{\mathrm{S}}\right)$, valores del Infrarrojo cercano (NIR), y valores del Índice de Vegetación de Diferencia Normalizada (NDVI), expresados en MegaPascales (MPa), ND de Radiancia NIR y Rango de NDVI (-1 a 1).

\begin{tabular}{lcccc}
\hline Tratamientos & & $\Psi_{\mathrm{S}}$ & NIR & NDVI \\
\hline \multirow{2}{*}{ Control } & $\mathrm{N}$ & 1020 & 1020 & 1020 \\
& Desv. típ. & 0,9874 & 456,228 & 0,44237 \\
& Mediana & $\mathbf{- 8 , 4}$ & $\mathbf{5 2 1 2}$ & $\mathbf{0 , 4 8 3 9 9 2}$ \\
\multirow{2}{*}{ RDCb } & $\mathrm{N}$ & 613 & 613 & 613 \\
& Desv. típ. & 2,2583 & 399,551 & 0,46613 \\
& Mediana & $\mathbf{- 1 7 , 2}$ & $\mathbf{4 9 5 4}$ & $\mathbf{0 , 4 4 6 5 3 7}$ \\
RDCa & $\mathrm{N}$ & 173 & 173 & 173 \\
& Desv. típ. & 1,6500 & 351,064 & 0,49405 \\
& Mediana & $\mathbf{- 1 9 , 8}$ & $\mathbf{4 9 5 0}$ & $\mathbf{0 , 4 4 4 9 8 3}$ \\
Total & N & 1806 & 1806 & 1806 \\
& Desv. típ. & 4,6823 & 449,980 & 0,48927 \\
& Mediana & $-9,8$ & 5094 & 0,468433 \\
\hline
\end{tabular}

\section{TABLA 2}

Comparación mediante el Test U de Mann-Whitney de los aportes de riego con el potencial hídrico $\left(\Psi_{\mathrm{S}}\right)$, los valores de las longitudes de onda del infrarrojo cercano (NIR) del espectro electromagnético y los valores Índice de Vegetación de Diferencia Normalizada (NDVI).

\begin{tabular}{lccc}
\hline & $\Psi_{\mathrm{S}}$ & NIR & NDVI \\
\hline U de Mann-Whitney & 0 & 12 & 13 \\
Sig. asintót. (bilateral) & 0 & 0,002 & 0,003 \\
Sig. exacta (bilateral) & $\mathbf{0}$ & $\mathbf{0 , 0 0 2}$ & $\mathbf{0 , 0 0 2}$ \\
Sig. exacta (unilateral) & 0 & 0,001 & 0,001 \\
\hline
\end{tabular}


Para medir el grado de dependencia que existe entre el potencial hídrico y las dos variables espectrales, y poder definir el grado de correlación existente entre las variables, se ha utilizado un procedimiento de correlación bivariada, mediante la cuantificación por los denominados coeficientes de correlación lineal de Pearson. Los valores de la tabla 3 nos muestran que se han encontrado correlaciones significativas entre $\Psi_{\text {s }}$ y NIR, con una correlación de tipo medio ( $\mathrm{r}=0.61)$, y de $\Psi_{\mathrm{S}}$ y NVDI, con una relación más estrecha $(\mathrm{r}=0.746)$, lo cual índica un mejor ajuste entre los valores del potencial hídrico y el NDVI que con los valores NIR. Existió también una correlación alta entre NIR y NDVI (r=0.752), pero en este caso dicha correlación era esperada, ya que ambas variables están basadas en datos de infrarrojo cercano.

\section{TABLA 3}

Grado de dependencia mediante cuantificación de correlación lineal de Pearson entre el potencial hídrico $\left(\Psi_{\mathrm{s}}\right)$ y las variables espectrales del infrarrojo cercano (NIR) y los valores Índice de Vegetación de Diferencia Normalizada (NDVI).** La correlación es significativa al nivel 0,01 (bilateral).

\begin{tabular}{llccc}
\hline & & $\Psi_{\mathrm{S}}$ & NIR & NDVI \\
\hline \multirow{3}{*}{$\Psi_{\mathrm{s}}$} & Corr. Pearson & 1 & $\mathbf{0 , 6 1 0 * *}$ & $\mathbf{0 , 7 4 6 * *}$ \\
& Signif. & & 0,003 & 0 \\
& $\mathrm{~N}$ & 21 & 21 & 21 \\
& Corr. de Pearson & $\mathbf{0 , 6 1 0 * *}$ & 1 & $\mathbf{0 , 7 5 2 * *}$ \\
\multirow{2}{*}{ NIR } & Signif. & 0,003 & & 0 \\
& N & 21 & 21 & 21 \\
& Corr. de Pearson & $\mathbf{0 , 7 4 6 * *}$ & $\mathbf{0 , 7 5 2 * *}$ & 1 \\
& Signif. & 0 & 0 & 21 \\
\hline
\end{tabular}

Con estas relaciones descritas entre estas variables se podrían determinar ciertos modelos de correspondencia entre los datos espectrales de imágenes obtenidas por sensores remotos y la perdida de vigor fenológico en los cultivos (MARTÍNEZ-FERNÁNDEZ y HERNÁNDEZ-SANTANA, 2011). La existencia de una estrecha relación entre la influencia del estrés hídrico en plantas y el cambio en sus procesos fisiológicos, queda reflejada en numerosos mecanismos de defensa que ponen en funcionamientos los cultivos para hacer frente a las limitaciones hídricas (KOZLOWSKI, 2002). Una de estas estrategias es el ajuste osmótico u osmorregulación, por el cual la planta aumenta de forma activa la concentración de solutos en su citoplasma para disminuir su potencial hídrico. De este modo, mantienen una diferencia importante entre el potencial hídrico de sus tejidos y el del suelo, aun cuando éste disminuye, por lo que el agua sigue incorporándose a la planta a favor de gradiente de potencial (CHAVES et al., 2003). Este proceso que sigue el mismo 
patrón en árboles frutales (ALARCÓN et al, 2000; ALARCÓN et al, 2003; BUENDÍA et al., 2008) nos permite correlacionar los valores del potencial hídrico del tallo de la hoja en la planta con los valores espectrales obtenidos en las imágenes (RIPPLE, 1986; KRISTON-VIZI et al., 2008).

\section{CONCLUSIONES}

(1) La variabilidad en la situación hídrica de los árboles, generada ante distintos aportes de agua mediante un riego diferenciado, fue medida en superficie por medio del potencial hídrico de tallo a mediodía ( $\left.\Psi_{\mathrm{s}}\right)$, y desde el aire por medio de la captación de imágenes medidas con una cámara multiesprectal que permitieron estimar los valores del espectro infrarrojo cercano (NIR) y del índice de vegetación de diferencia normalizada (NDVI).

(2) Los tres índices considerados fueron sensibles al grado de déficit hídrico generado en los árboles, según los análisis estadísticos llevados a cabo por medio de la prueba de Mann-Whitney y considerando las diferencias existentes entre árboles control y deficitarios.

(3) Los tres índices considerados presentaron significativos valores de correlación cuando fueron comparados por correlación bivariada, obteniéndose el mejor grado de dependencia el aportado por el potencial hídrico de tallo ( $\left.\Psi_{\mathrm{S}}\right)$ con NVDI.

(4) Las correlaciones extraídas en los análisis estadísticos en este trabajo, pueden ser válidas para determinar comportamientos fenológicos de los cultivos tales como la disminución del vigor fotosintético de las hojas. Esta correspondencia entre los datos espectrales de imágenes obtenidas por sensores remotos y las restricciones hídricas del riego deficitario, podrían extrapolarse a mayores áreas de cultivo gracias a la capacidad de la teledetección de la obtención masiva de datos.

\section{AGRADECIMIENTOS}

Este ensayo ha sido financiado por el proyecto EU del programa SUDOE TeleriegSOE1/P2/E082.

\section{REFERENCIAS BIBLIOGRÁFICAS}

AGENCIA ESTATAL DE METEOROLOGÍA (AEMET). (Fecha de consulta: 23-05-2010). Disponible en: http://www.aemet.es/

AGUSTÍ, M. (2003): Citricultura. Madrid, Ediciones Mundi-Prensa.

ALARCÓN, J.J., DOMINGO, R., GREEN, S.R., SÁNCHEZ-BLANCO, M.J., RODRÍGUEZ, P., TORRECILLAS, A. (2000): «Sap flow as an indicator of transpiration and the water status of young apricot trees». Plant and Soil, Volume 227, 1-2. Pp. 77-85.

ALARCÓN, J.J. , DOMINGO, R., GREEN, S.R., NICOLÁS, E., TORRECILLAS, A. (2003): «Estimation of hydraulic conductance within field-grown apricot using sap flow measurements». Plant and Soil, Volume 251, 1. Pp. 125-135.

ALARCÓN, J.J., ORTUÑO, M.F., NICOLÁS, E., NAVARRO, A. AND TORRECILLAS, A. (2006): "Improving water-use efficiency of young lemon trees by shading with aluminised-plastic nets». Agricultural Water Management, Volume 82, 3. Pp. 387-398. 
ARCAS, N. Y HERNÁNDEZ, M. (2010): Caso Alimer: Ganar competitividad a través de la fusión; Munuera Alemán, J. L., coord.: Casos de éxito de las empresas murcianas. Madrid, ESIC. Pp. 273-286.

ARAUS, J.L. (2004): «The problems of sustainable water use in the Mediterranean and research requirements agriculture». Annals of Applied Biology, v. 144, n3, pp. 259-272.

BANNARI, A., MORIN, D., BONN, F. AND HUETE, A. (1995): «A review of vegetation indices». Remote Sensing Reviews. 13. Pp. 95-120.

BERNI, J.A.J., ZARCO-TEJADA, P.J., SUÁREZ, L., FERERES, E. (2009): «Thermal and Narrowband Multispectral Remote Sensing for Vegetation Monitoring From an Unmanned Aerial Vehicle». IEEE Transactions on Geoscience and Remote Sensing 47, 3. Pp. 722-738.

BLANCO-GUTIÉRREZ, I., VARELA-ORTEGA, C., PURKEY, D.R. (2013): «Integrated assessment of policy interventions for promoting sustainable irrigation in semi-arid environments: A hydro-economic modeling approach». Journal of Environmental Management. Volume 128, 5, Pp. 144-160

BUENDÍA, B., ALLENDE, A., NICOLÁS, E., ALARCÓN, J.J., GIL, M.I. (2008): «Effect of regulated deficit irrigation and crop load on the antioxidant compounds of peaches». Journal of Agricultural and Food Chemistry, Volume 56, 10. Pp. 3601-3608.

CHALMERS, D.J. (1989): «A physiological examination of regulated deficit irrigation». New Zealand Agricultural Science, Volume 23. Pp. 44-48.

CHAVES, M.M., MAROCO, J.P., PEREIRA, J.S. (2003): «Understanding plant responses to drought - From genes to the whole plant». Functional Plant Biology, 30, 3. Pp. 239-264.

CHUVIECO, E. (2002): Teledetección Ambiental: La Observación de la Tierra Desde el Espacio. Ed. Ariel.

CHUVIECO, E., SALAS, F. J., AGUADO, I., COCERO, D. Y RIAÑO, D. (2001): «Estimación del estado hídrico de la vegetación a partir de sensores de alta y baja resolución». GeoFocus, nº 1 . Pp. 1-16.

CRIPPEN, R. E. (1990): «Calculating the Vegetation Index Faster». Remote Sensing of Environment, 34. Pp. 71-73.

DZIKITI, S., VERREYNNE, J.S., STUCKENS, J., STREVER,A., VERSTRAETEN, W.W., SWENNEN, R., COPPIN, P. (2010): «Determining the water status of Satsuma mandarin trees [Citrus Unshiu Marcovitch] using spectral indices and by combining hyperspectral and physiological data». Agricultural and Forest Meteorology 150 , 3. Pp. 369-379.

FAO-UNESCO. (1974): Soil Map of the world. Vol. I: Legend.

FERERES, E. y SORIANO, M.A. (2007): Deficit irrigation for reducing agricultural water use. Journal of Experimental Botany, 58 , 2. Pp. 147-159

GARRIDO, A., MARTÍNEZ-SANTOS, P., LLAMAS, M.R. (2006): «Groundwater irrigation and its implications for water policy in semiarid countries: The Spanish experience». Hydrogeology Journal. Volume 14, Issue 3, Pp. 340-349.

GILABERT, M.A., GONZÁLEZ-PIQUERAS, J. AND GARCÍA-HARO, J. (1997): «Acerca de los Índices de Vegetación». Revista de Teledetección, No. 8.

GINESTAR, C. Y CASTEL, J.R. (1996): «Responses of young clementine citrus trees to water stress during different phenological periods». Journal of Horticulture Science, 71, 4. Pp. 551-559. 
GOWARD, S.N.; HUEMMRICH, K.F. (1992): «Vegetation canopy PAR absorbance and the Normalize Difference Vegetation Index: An assessment using the SAIL model». Remote Sensing of Environment, 39. Pp. 119-140.

HSIAO, T.C. (1990): «Measurements of plants water status. In: Irrigation of Agricultural Crops». Agronomy Monograph, 30. Pp. 243-279.

KIM, Y., GLENN, D.M., PARK, J., NGUGI, H.K., LEHMAN, B.L. (2011): «Hyperspectral image analysis for water stress detection of apple trees». Computers and Electronics in Agriculture 77, 2. Pp. 155-160.

KOGAN, F.N. (1997): «Global Drought Watch from Space». Bulletin of the American Meteorological Society, 78, 4. Pp. 621-636.

KOZLOWSKI, T.T., PALLARDY, S.G. (2002): «Acclimation and adaptive responses of woody plants to environmental stresses». Botanical Review, 68, 2. Pp. 270-334.

KRISTON-VIZI, J., UMEDA, M., MIYAMOTO, K. (2008): «Assessment of the water status of mandarin and peach canopies using visible multispectral imagery». Biosystems Engineering, 100, 3. Pp. 338-345.

LOUSSERT, R. (1992): Los Agrios. Madrid, Ediciones Mundi-Prensa.

LUCDEME. Proyecto de Lucha contra la desertificación en el Mediterráneo. (1986): Mapa de Suelos escala 1:100.000, Murcia - 934. Ministerio de Agricultura, Pesca y Alimentación, ICONA, Universidad de Murcia, Murcia.

LYCHAK, O Y JAREMY, M . (2000): «Influence of possible ways of remote sensing data and digital data nonlinear transformation on the results of unsupervised classification». Conference on Applications of Digital Image Processing XXIII. San Diego, EE.UU.

MARTÍNEZ-BELTRÁN, C., JOCHUM, M.A.O., CALERA, A., MELIÁ, J. (2009): «Multisensor comparison of NDVI for a semi-arid environment in Spain». International Journal of Remote Sensing 30. Pp. 1355-1384.

MARTÍNEZ-FERNÁNDEZ, J.; HERNÁNDEZ-SANTANA, V. (2011): «Soil water reserve estimation and vegetation relationships in a Mediterranean subhumid forested catchment». Hydrology Research 43. Pp. 167-178.

ORTUÑO, M.F., ALARCÓN, J.J., NICOLÁS, E. AND TORRECILLAS, A. (2004): «Interpreting trunk diameter changes in young lemon tres under déficit irrigation». Plant Science, 167, 2. Pp. 275-280.

RIPPLE, W.J. (1986): «Spectral reflectance relationships to leaf water stress». Photogrammetric Engineering \& Remote Sensing, 52, 10. Pp. 1669-1675.

ROUSE, J.W.; HAAS, R.H.; SCHELL, J.A.; DEERING, D.W.; HARLAN, J.C. (1974): «Monitoring the vernal advancement of retrogradation of natural vegetation». NASA/ GSFC Type III Final report. Maryland. Pp. 371.

RUIZ-SÁNCHEZ, M.C., DOMINGO, R., SAVE, R., BIEL, C. AND TORRECILLAS, A. (1997): «Effects of water stress and rewatering on leaf wáter relations of lemon plants». Biologia Plantarum, 39, 4. Pp. 623-631.

SAVE, R., BIEL, C., DOMINGO, R., RUIZ SÁNCHEZ, M.C. AND TORRECILLAS, A. (1995): «Some Physiological and Morphological-Characteristics of Citrus Plants for Drought Resistance». Plant Science, 110, 2. Pp. 167-172.

STAGAKIS, S.; GONZÁLEZ-DUGO, V.; CID, P.; GUILLÉN-CLIMENT, M. L.; ZARCOTEJADA, P. J. (2012): «Monitoring water stress and fruit quality in an orange orchard 
under regulated deficit irrigation using narrow-band structural and physiological remote sensing indice». ISPRS Journal of Photogrammetry and Remote Sensing, 71. Pp. 47-61. SUÁREZ, L., ZARCO-TEJADA, P.J., GONZÁLEZ-DUGO, V., BERNI, J.A.J., SAGARDOY, R., MORALES, F., FERERES, E. (2010): «Detecting water stress effects on fruit quality in orchards with time-series PRI airborne imagery». Remote Sensing of Environment 114, 2. Pp. 286-298.

VANDEN HEUVEL, R.M. (1996): «The promise of precision agriculture». Journal of Soil and Water Conservation, 51, 1. Pp. 38. 